\title{
FREQUENCY OF TRAP CLOSURE AND CAPTURE OF PREY BY DIONAEA MUSCIPULA IN THE FIELD
}

\author{
StePHEN E. WiLliams $・$ Emeritus Professor $・$ Lebanon Valley College $・$ Annville $・$ Pennsylvania \\ 17003・USA・williams@lvc.edu \\ BILL SCHOLL・Liberty County・Florida 32321・USA・scholljunk@gmail.com
}

\begin{abstract}
Measurements in the field on dry days indicate that when the traps of Dionaea muscipula (Venus' flytraps) close, they capture prey about $40 \%$ of the time at one site and $44 \%$ at another. The $60 \%$ and $56 \%$ of traps that close but do not catch prey reach their maximum closure on average in about 10 minutes, begin reopening in about 7 hours and completely reopen in 22 hours. Traps that have captured prey tighten along their rims, sealing the lobes and forcing the margins outward into a "narrowed" condition that takes 4 to 6 hours to achieve. Reopening of narrowed traps begins, on average, after 9.2 days and they are fully open in 12.8 days. A few traps that capture large prey never reopen.
\end{abstract}

In dry weather closure frequency of traps capable of closure was low, averaging 3.5\%/day at one site and 3.1\%/day at another. At both sites the capture frequency of traps capable of closing was only $1.4 \%$ /day. The low frequency of capture, and high percentage of closures that result in capture, indicate that the low capture frequency is due to a low number potential prey entering and triggering the trap rather than a low efficiency of capturing the prey that enters.

Traps that close, but fail to capture prey, have been observed to be triggered by insects, spiders, snails, and even toads that were far too large to be captured. A few captured beetles have been observed to force their way out of trap lobes.

Dionaea traps capture prey at all hours of the day. The capture frequencies are variable and are not significantly higher in any specific time period. Both nocturnal and diurnal animals were captured.

\section{Introduction}

Dionaea muscipula has an elaborate snap trap that has attracted nearly universal attention. Numerous popular descriptions describe flies being attracted to copious nectar secreted from glands along the edge of the trap but with no evidence cited. Adrian Slack (1980) states "Many insect visitors are undoubtedly attracted to the trap by the scent of the nectar supply, which is probably further advertised by the red coloration of the lobes, but a large number are casual visitors which are not nectar feeders." These ideas probably started with very convincing videos from the $\mathrm{BBC}$, which have been reviewed by Williams and Hartmeyer (2017). While the actual frequency of capture is not mentioned in these sources, the suggested effectiveness of the attractants infers that captures should be frequent.

Actually, the limited observations that have been made suggest that prey capture is relatively infrequent (Williams \& Hartmeyer 2017; Lichtner \& Williams 1977) and a visit to their native habitat in North Carolina on a sunny day can easily confirm that most of the traps are open although they are capable of rapidly snapping shut if stimulated. Williams (1980), describing earlier measurements he made with Frank Lichtner, says that on a day with no rain $2.0 \%$ of 201 traps closed without capturing prey and $3.0 \%$ of traps captured prey. At this frequency of capture a typical trap would catch prey about once every 50 days. This single measurement made in the plant's natural habitat, 
and similar measurements made by Williams and Hartmeyer (2017) on greenhouse and garden plants should be supplemented by more observations of plants in the wild.

The low capture frequencies of Dionaea could be due either to a small number of visitors to the traps, to a small number of the visitors triggering the traps, to ineffectiveness of triggered traps in capturing prey, or to a combination of these factors.

We have observed trap closures and captures by Dionaea plants growing in a stand grown from seed broadcast on the gulf coastal savannah of Florida 15 to 17 years ago (Site 2) and a second smaller population that descends from about six Dionaea plants transplanted about 15 years ago (Site 3). These plants descend from seeds originally harvested in the Carolinas (Williams \& Scholl 2021a).

Our object is to measure closure frequencies and capture frequencies of Dionaea traps as well as rates of reopening of traps in a wild area similar to their native habitat. We also observed actual captures in the field and the factors that cause closure of the traps that fail to capture prey.

\section{Methods}

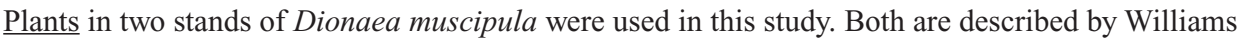
and Scholl (2021a). One is a wet site near a small stand of Titi trees (Clitonia monophylla) that have covered the ground with leaf litter. Sphagnum is growing among the Dionaea plants (Site 3). The second is the neighboring Florida population that originated from seed, descended from plants from the Carolinas broadcast 15 to 17 years ago (Site 2). These are growing among scattered tall pines in a grassy area typical of the coastal savannah.

Photography and videography were done using a Bushnell NatureView camera mounted horizontally on a TopView Quick-Mount camera stand, above the plants being observed. The camera recorded a $25 \times 35 \mathrm{~cm}$ field of view as still photographs and a $15 \times 24.5 \mathrm{~cm}$ field of view as video. Every 5 minutes a photograph was taken, immediately followed by 1-minute video. The photographs were overlain with a grid and sequentially placed in a slideshow, which was scanned to observe the times of trap closure. Videos recorded after trap closure were then observed to determine if the traps reopened or narrowed from the capture of prey. When possible the cause of trap closure was also observed. Details of the camera methods are described by Williams and Scholl (2021a).

Method of measuring frequency of trap closure was the same as used by Williams and Scholl (2021b).

Trap closure occurs in a fraction of a second and is the flipping of the trap from the open position to the position in which the bristles along the edge of the leaf cross each other and only small gaps exist along the rim of the trap.

Trap narrowing (Fig. 1) occurs within minutes of the capture of prey. Mechanical stimulation from the struggling prey and later chemical stimulation from decomposing prey causes trap narrowing (Williams \& Pickard 1980). Narrowed trap's lobes close tightly together, sealing along a smooth area just inside the edge of each trap lobe. The bristles along the edge of a narrowed trap are forced outward giving a narrowed trap a distinctive look. Narrowed traps can be used as a way to identify closed traps that have captured prey.

Active traps, as used in this study, refers to traps that are capable of closing.

Frequency of trap closure per day = closures/active-traps/day.

Frequency of capture per day $=$ narrowed-traps/active-traps/day.

Frequency of closure without capture $=($ closures/active-traps/day $)$ - (narrowed-traps/active-traps/day). 

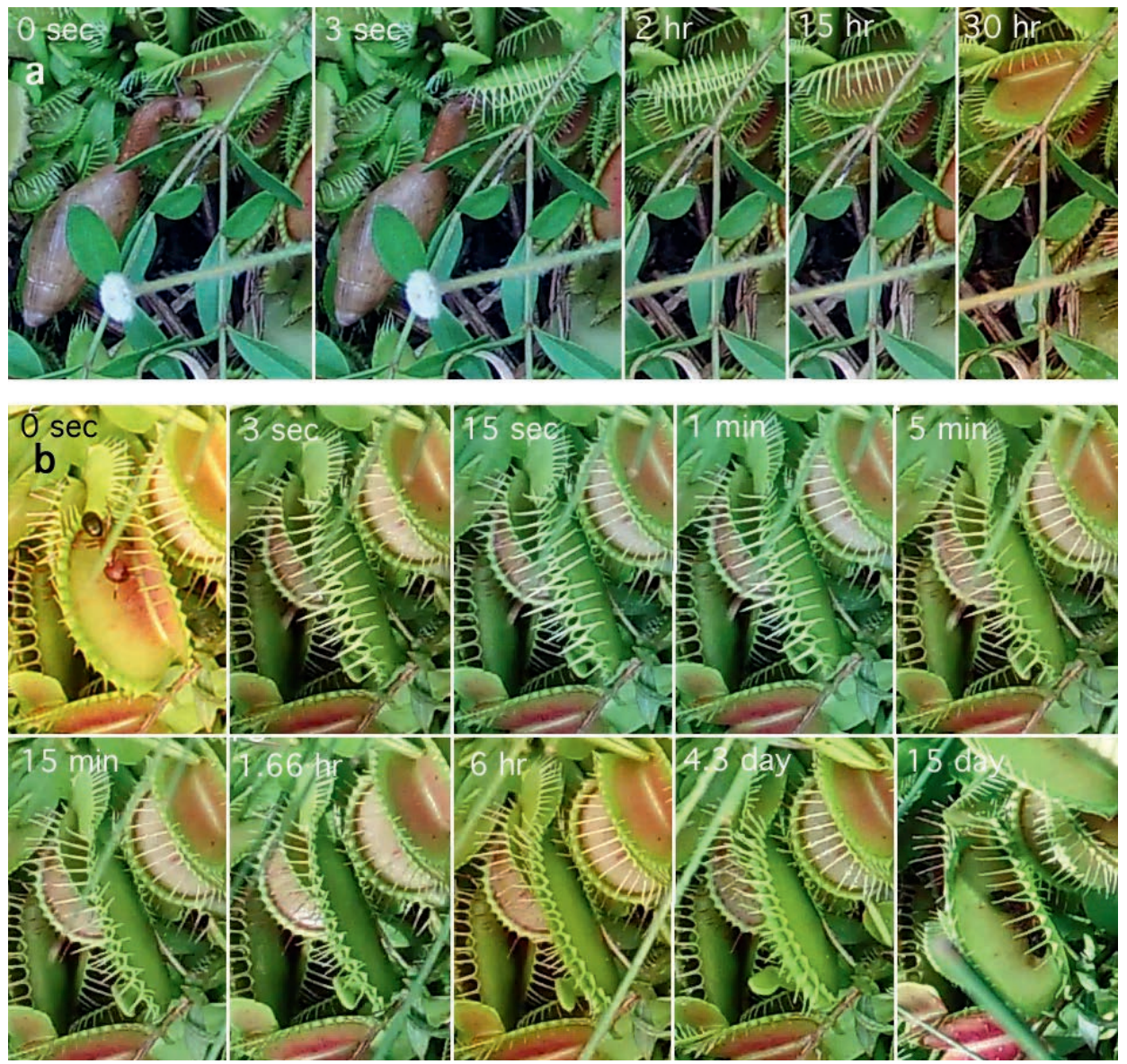

Figure 1: a. Closure without capture. A trap set off by a large snail. The trap is stimulated to close quickly, the snail pulls its head free and the trap opens after dark in 15 hours and fully opened by 30 hours. b. Capture and trap narrowing. A large ant triggers the trap to close. The ant's struggles stimulate the trap lobes to press together tightly sealing along their rims in about 5 minutes. They continue to press more tightly together for 6 hours causing their rims to flex outward in the "narrowed" position. After about two weeks the trap is fully reopened, revealing the undigested exoskeleton of the ant.

Measuring frequency of closure and capture at different times of day

Trap closures and captures were tallied in eight, three-hour periods: Early morning (03-06), Morning (06-09), Late morning (09-12), Early afternoon (12-15), Late afternoon (15-18), Evening (1821), Night (21-00), Late night (00-03). Times are EST on a 24-hour clock.

\section{Measuring reopening time}

Reopening time of a trap was measured by subtracting the time when the trap was closed from the time when the trap was fully reopened. Both narrowed traps, that have captured prey, and closed traps, that have not captured prey, were measured in the same way. We also determined the time between trap closure and the time when the trap first began to move its lobes apart. 
Measuring proportion of traps that are open and in other states

A $15 \mathrm{~cm}$ by $24 \mathrm{~cm}$ area was observed during dry weather on five dates between 7/17/20 and 10/10/20 and the proportion of traps in open, closed, narrowed, and reopening states was measured. The traps too young and too old to be active were disregarded but the traps which had captured prey and were in a narrowed state were included in "total traps" for this measurement. Traps in the reopening state can be reopening from the narrowed state or the closed state.

\section{Trap Narrowing}

\section{Results}

At least two stimuli to a trigger hair, or one stimulus to each of two hairs of a Dionaea trap within a period of 20 seconds will cause the lobes of the trap to come together, closing it (Brown \& Sharp 1910; Fig. 1a). Additional stimuli will cause the lobes of the trap to tighten further and press against each other. When prey is captured, its struggles deliver numerous stimuli to the trap's trigger hairs, each of which causes the lobes to press more tightly against each other, ultimately pressing so hard that the edge of the lobes are forced outward and the lobes are sealed tightly along a smooth region just inside their outer edges (Lichtner \& Williams 1977; Fig. 1b). Traps with this appearance are called "narrowed" (Lloyd 1942) and form a digestive cavity, which has been called a "green stomach" (Scherzer et al. 2015).

Traps stimulated to close which have no further stimulation do not close tightly and reach their maximum closure in about 10 minutes. They begin reopening in 7 hours and are completely reopened in 22 hours.

Traps that have captured prey receive additional mechanical stimulation from the prey's attempt to escape, and later by chemical stimulation during the prey's decomposition (Lichtner \& Williams 1977). This further stimulation causes the trap to tighten, sealing its lobes along a smooth strip along each lobe resulting the trap reaching a narrowed state. The initial trap closure typically takes less than a second but it takes traps 4 to 6 hours to become fully narrowed and days to weeks to reopen (Table 1).

The traps that failed to capture prey began to open in an average of 0.29 days and were fully open in an average of 0.90 days (Fig. 1a, Table 1). Five traps that have captured prey and narrowed began to reopen in an average of 9.2 days and were fully open in 12.8 days (Fig. 2a, Table 1), but when large prey is captured, or when the trap is older, opening can take much longer and may not occur at all (Fig. 2 in Williams \& Scholl 2021a). Traps that have captured prey take on average about fourteen times as long to begin to reopen and seven times as long to fully reopen. Reopening of traps that have not captured prey is completed in about a day while those that have captured prey and digest it take weeks to reopen.

\begin{tabular}{|c|c|c|}
\hline & \multicolumn{2}{|c|}{ Reopening Times (days) } \\
\hline & No Capture & Capture (narrowed) \\
\hline Number of Samples & 10 & 5 \\
\hline Begin reopening (mean \pm SEM) & $0.29 \pm 0.027$ & $9.2 \pm 1.47$ \\
\hline Fully reopened (mean \pm SEM) & $0.90 \pm 0.038$ & $12.8 \pm 1.92$ \\
\hline
\end{tabular}




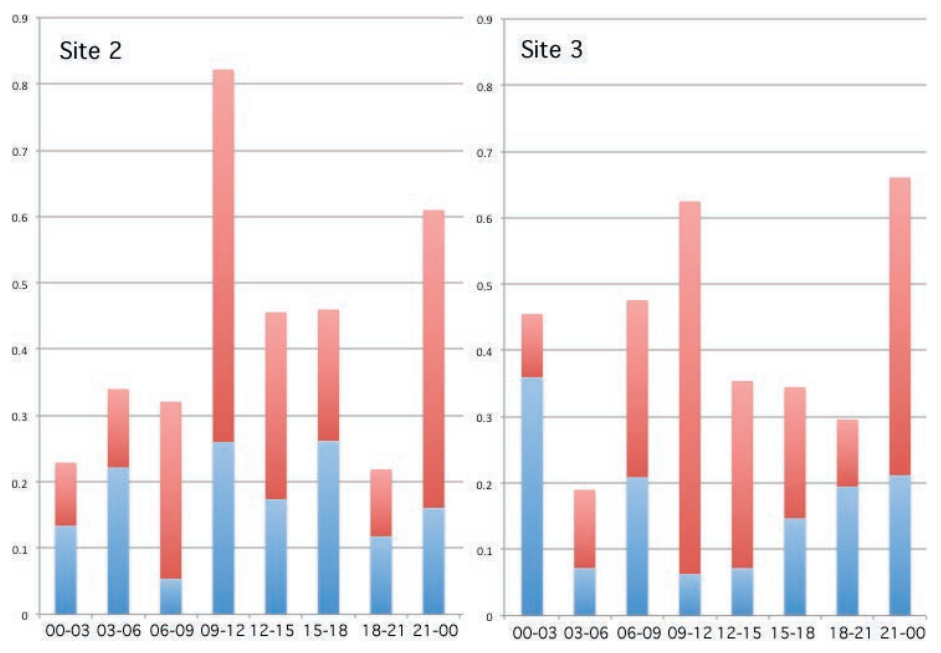

Figure 2: a. Ten-day average of captures and closures during 8 time periods at Site 2. Blue bars show the capture frequency. Red bars show the frequency of closure where there was no capture; the total bar height is the frequency of closures. The times at the base of the bars are 24 hours EST. The units on the vertical axis are closures/active trap/3 hours. b. Five-day average captures and closures during 8 time periods at Site 3. Blue bars show the capture frequency. Red bars show the frequency of closure where there was no capture and the total bar height is the frequency of closures. The times at the base of the bars are 24 hours EST. The units on the vertical axis are closures/active trap/3 hours.

Closure and capture frequencies

Frequencies of closure and prey capture of traps of two stands of Dionaea growing in the Gulf coastal plain in the Florida panhandle were determined from time-lapse photography. Site 2 is typical grassy coastal savannah amidst mature pine trees while Site 3 is a wetter area with Sphagnum near a stream within several meters of a stand of Titi trees (Clitonia monophylla).

The average frequency of prey capture, during 10 days, for traps at Site 2 was $1.39 \%$ of active traps/day and at Site 3 the average frequency, during 5 days, was $1.33 \%$ of active traps/day (Table 2).

Total trap closure, including closures that captured prey, had an average frequency of $3.45 \%$ of active traps/day at Site 2 and an average of $3.06 \%$ of active traps/day at Site 3 . About $40 \%$ of the closures at both sites resulted in prey capture (Table 2). The highest capture frequency per day was $4.9 \%$ of active traps at Site 2 . On that day $7.0 \%$ of the active traps closed and $70 \%$ of the closures resulted in captures.

The capture frequency of Dionaea in the field is low. The average frequency during the 15 days of observation was less than $1.4 \%$ of active traps capturing prey in a 24-hour period but when traps are triggered to close, they capture prey over $40 \%$ of the time. On the most successful day, $70 \%$ of closures resulted in prey capture. Traps are inefficient in luring prey to trigger the trap but are relatively effective in capturing the prey that does trigger trap closure. Some insects, spiders, snails, and even toads that are all far too large to be captured often trigger traps that close but fail to capture 
Table 2. Trap closures per active-trap per day and closures with and without capture at two different sites.

\begin{tabular}{|l|c|c|}
\hline \multicolumn{1}{|c|}{ Location } & Site 2 & Site 3 \\
\hline Number of days sampled & 10 & 5 \\
\hline Percent total closures (Clos/ActTr/Day) & 3.45 & 3.06 \\
\hline Percent close no-capture (Clos/ActTr/Day) & 2.07 & 1.73 \\
\hline Percent captures (Captures/ActTr/Day) & 1.39 & 1.33 \\
\hline Percent closed that captured & 40.3 & 43.5 \\
\hline
\end{tabular}

prey. Both animals that are captured and those too large or strong for traps to capture blunder into traps. Their behavior is not what would be expected of an animal being lured by an attractant.

Comparison with previous research

The capture frequencies measured at both Florida sites were about half that reported in North Carolina in June by Williams (1980). Closure frequencies at both sites were lower than those reported in North Carolina. There were only one third as many closures at Site 2 and Site 3 as were measured in the Carolinas. The capture frequencies at Site 2 are one third to one half as many as in the earlier study in North Carolina, but of the traps that closed in the North Carolina study only $29.6 \%$ captured prey as opposed to greater than $40 \%$ in Florida.

Proportion of traps in open, narrowed, closed, and reopening states in summer and early fall.

When viewed in the field either in the Carolinas or in the Florida panhandle most traps appear to be open. Actual measurements of the proportion of traps that are open confirm this impression (Table 3). Eighty-three to ninety percent of the traps were open at each of five observation dates. Six to twelve percent of the traps had narrowed after capturing prey. Since traps that are narrowed often remain closed for a week or more it is not surprising that they are the largest group of traps that are not open. Less than $2 \%$ of the traps are closed and $2.4 \%$ to $5 \%$ of the traps are reopening either from the closed (with no capture) or the narrowed stage.

Table 3. Proportion of traps in various stages at noon EST during the summer and early fall of 2020. Total traps = active traps + narrowed traps. Reopening traps are reopening either from the narrowed stage or the closed stage. Traps were at Site 2 in a $15 \times 24 \mathrm{~cm}$ area.

\begin{tabular}{|l|c|c|c|c|c|}
\hline \multicolumn{1}{|c|}{ Date } & $\begin{array}{c}\text { Traps } \\
\text { Total }\end{array}$ & $\begin{array}{c}\text { Percent } \\
\text { Narrowed }\end{array}$ & $\begin{array}{c}\text { Percent } \\
\text { Closed }\end{array}$ & $\begin{array}{c}\text { Percent } \\
\text { Reopening }\end{array}$ & $\begin{array}{c}\text { Percent } \\
\text { Open }\end{array}$ \\
\hline 17-Jul & 135 & 5.9 & 1.5 & 3.0 & 89.6 \\
\hline 1-Aug & 142 & 12.0 & 2.1 & 2.8 & 83.1 \\
\hline 21-Aug & 170 & 11.2 & 0.0 & 4.0 & 84.7 \\
\hline 18-Sep & 164 & 11.6 & 1.2 & 2.4 & 84.8 \\
\hline 10-Oct & 211 & 7.1 & 1.9 & 4.7 & 86.3 \\
\hline
\end{tabular}


Closure and Capture frequency at different times of day

Closure and capture frequencies were measured for ten days at Site 2 and for five days at Site 3 during eight 3-hour time periods. Dionaea traps capture prey at all hours of the day. The capture frequencies were variable and were not significantly higher in any specific period. Closure of traps that did not capture prey also had variable frequencies with no relation to time of day (Fig. 2). Traps at Site 3 captured a few more prey at night. On this site we observed activity by nocturnal spiders that also were visible as remains in reopened traps. It is likely that any difference in frequency of closure or capture is due to the relative prevalence of nocturnal or diurnal prey instead of characteristics of the traps.

Interaction of traps with animals

Prey that were observed being captured included ants, gnats, spiders, and grasshoppers.

Ants, which typically compose about one third of the prey of Dionaea (Williams \& Hartmeyer 2017), were observed being captured when scrambling from one place to another passing through several traps in the process. Occasionally they trigger a small or medium trap as they pass through and are captured. Most of the observed ants are small but a large $(10 \mathrm{~mm})$ ant was observed while in the center of one of the large traps away from the peripheral glands (Fig. 1).

Gnats often walk along the edge of traps pausing at the peripheral glands (aka "alluring glands"), that appear to act as nectaries (Fig. 3a). When behaving in this way they almost always move along the edge of the leaf, only rarely moving away from it. They usually walk from leaf to leaf but sometimes fly to an adjacent leaf. Gnats are only a small part of prey captured by Dionaea but they are sometimes captured when suddenly landing on a leaf (Fig. 3b). We observed small gnats and ants working the peripheral glands with their mouthparts in the same way as those reported by Jones (1923) and Hartmeyer et al. (2019). Gnats and ants feeding along the rim were not observed to cause trap closure even though both occasionally walked across the traps near the trigger hairs.

Peripheral glands are located along the rim of each trap lobe just outside the smooth area where the lobes seal during narrowing. Structurally they are nearly identical to the digestive glands
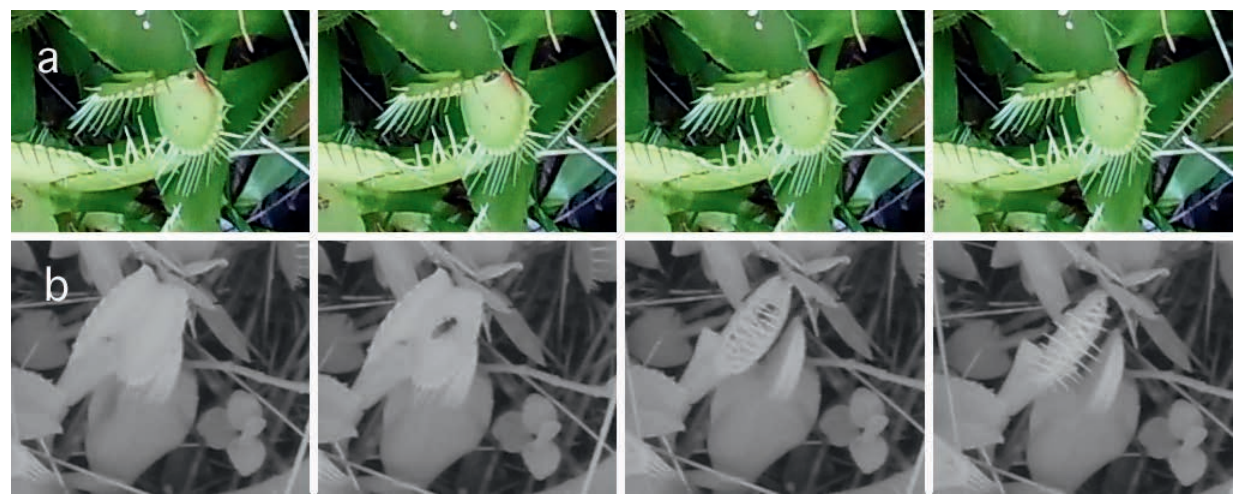

Figure 3: a. A $2.2 \mathrm{~mm}$ gnat gradually moves along the rim of the upper lobe of a trap pausing for extended periods, as it appears to feed on nectar from the peripheral glands. The sequence of video frames covers 30 seconds. b. A $3.5 \mathrm{~mm}$ gnat lands on a trap, brushes past the trigger hairs and is captured. Video frames from $3 \mathrm{a}$ and $3 \mathrm{~b}$ are at the same magnification. $3 \mathrm{~b}$ is black and white because the light intensity dropped below that required for color images. 
that cover the inner surface of the lobes except for their smaller size and lower pigmentation. First described by Jones (1928) as "nectar glands?" that attracted small ants that did not set off the trap. They were named alluring glands by Lloyd (1942). Juniper et al. (1989) later called them the "peripheral glands". The evidence for their role as nectaries were Jones' observations and videos of captures made by the BBC (reviewed by Williams \& Hartmeyer 2017; Hartmeyer et al. 2019). The name "alluring glands" suggest that these glands play a major role in attracting prey into the trap but there is little evidence that they do. We adopted Juniper's term "peripheral glands" because it describes their position without designating a function.

Spiders typically compose up to a third of the prey of Dionaea (Williams \& Hartmeyer 2017). Almost all of the spiders observed on traps were nocturnal wolf spiders. The smaller ones that are captured sometimes appear to be hiding in traps to avoid larger wolf spiders which have been observed stalking them. On one occasion a small spider was observed moving through the trap without being captured.

Grasshoppers are reported by Lichtner and Williams (1977) to compose about $15 \%$ of the prey they observed captured in a study conducted in June. These were probably smaller nymphs. Other studies, done at different times of year, report relatively few grasshoppers captured. A grasshopper, of medium size, was captured by a large trap (Fig. 4b) while eating its way along a grass blade that passed through the center of a trap. The leaf closed around it but the grasshopper was too large for the trap to form a seal between the two lobes. Later small ants were attracted to the leaf, some of which were captured by neighboring traps.

Potential prey that trigger traps then escape capture include beetles of both the weevil type (Fig. 3 in Williams \& Scholl 2021) and the scarab type. Both are very strong and have a smooth hard

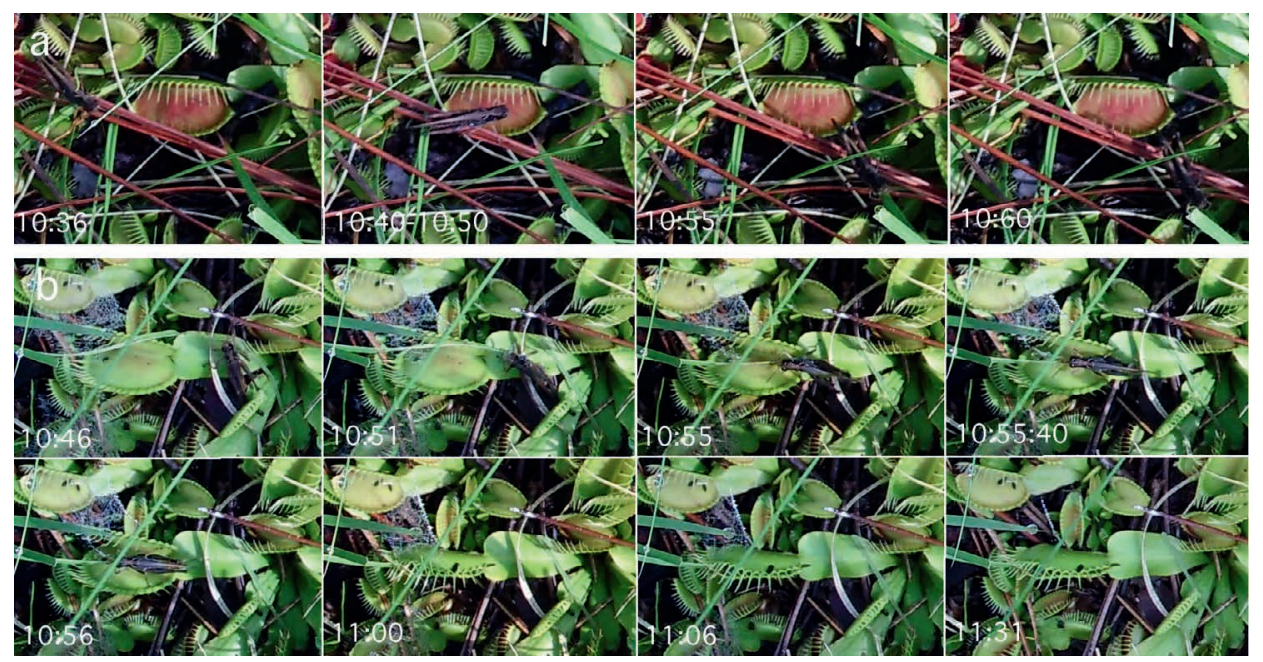

Figure 4: a. A grasshopper enters a Dionaea trap, remains there perfectly still for over 10 minutes and walks away without springing the trap. b. Simultaneously, a second grasshopper, feeding on a grass blade that passes through a trap, walks over the trigger hairs and triggers the trap resulting in its' closing and narrowing. The relatively large grasshopper prevents the trap from sealing properly ultimately drawing ants to the partially closed trap. 
carapace. In addition, the weevils have a pointed head and thorax that easily slips through the lobes of the trap. These insects can be cleanly captured, completely surrounded by the lobes of the trap for several minutes and slip out of its grasp and emerge in as little time as one minute, although one weevil struggled for 4 hours before breaking free. One weevil was trapped again and again setting off two additional traps within 5 minutes of its escape from the original trap.

Potential prey that enter traps and do not trigger them include ants, gnats, grasshoppers, and spiders. All have been observed entering traps and moving about without triggering closure. The ants and gnats avoid capture in the larger traps by keeping to the rim of medium and large traps where they are attracted away from the trigger hairs and toward the peripheral glands (Hartmeyer et al. 2019). In smaller traps they would easily reach the trigger hairs. It is possible that they serve as prey for smaller traps. On the same video as the grasshopper captured by a large trap (Fig. 4b) a second, nearly identical, grasshopper sat in the center of a large trap for over $10 \mathrm{~min}$ before exiting it without setting off the trap (Fig. 4a). Spiders sometimes crawl through traps without triggering them.

In preparing for this study, we saw a great many traps on plants that were cultivated in a terrarium catch ants and crickets in awkward ways. By contrast, in the captures we observed in the field, the trap lobes always surrounded the insect when triggered. Some beetles were able to escape after a clean capture.

Animals too large to capture that trigger traps were observed setting off traps. These include large grasshoppers, crickets and spiders, a narrow-mouthed toad, and a snail. These set off traps by stepping on them and sometimes by probing them. As many as three traps within 5 min were observed to be triggered by animals passing through the field of view. Small snakes, lizards, and an oak toad that are capable of triggering traps in this way have also passed through the field of view.

Large nocturnal wolf spiders often walk into the field of view and step on traps while they wait for prey, occasionally triggering them (Fig. 5). During the day, large grasshoppers grazing on grass also occasionally trigger traps. An unexpected visitor was a land snail that stuck its head into two
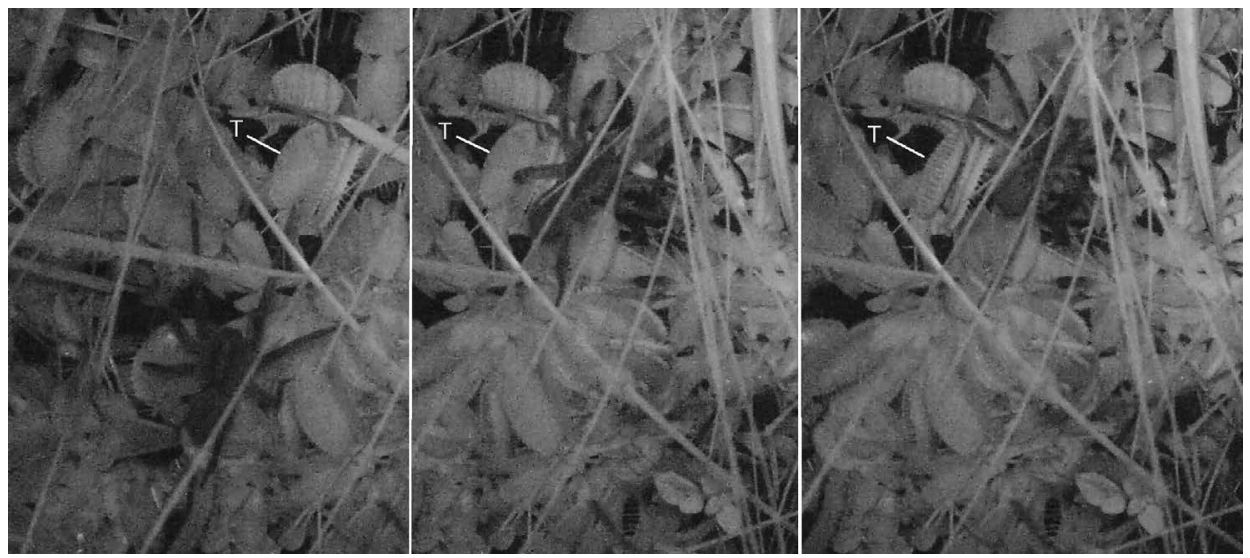

Figure 5: A large wolf spider steps on a trap "T" as it walks through a cluster of Dionaea plants just after midnight. Trap "T" is triggered but it has no chance of catching such a large spider. 
traps, triggering their closure before pulling its head free from the closed trap (Fig. 1a). Many of the $60 \%$ of traps that close without capturing prey may be triggered in this way.

Efficient capture of the few potential prey that enter a trap

When Dionaea traps are triggered to close, about $40 \%$ of the closed traps capture prey yet fewer than $3.5 \%$ of the traps capable of capturing prey make captures on an average day. The capture mechanism is remarkably effective in catching visitors that trigger the trap but it does very poorly at attracting prey, suggesting that any postulated attractants the traps produce to attract prey are ineffective or absent. Yet, the peripheral glands do seem to attract small ants and gnats that move about the rim of the traps without triggering them. What is the function of the peripheral glands if they do not contribute to the capture of prey? In this study, we observed medium to large traps of mature plants. It is possible that in seedlings and the small traps of immature plants, the gnats and small ants provide important nutrients.

\section{Summary}

In a population of Dionaea in the field, either in the Carolinas or in Florida, most of the traps are open at any given time, allowing maximum exposure of the leaf surface to the sun. Capture and digestion of prey have been shown to supply mineral nutrients to the plants (Adamec 1977). The Dionaea plants appeared to be very healthy despite the fact that prey capture by individual traps is very low, indicating that a frequency of capture of $1.4 \%$ of the active traps per day in a population is adequate to provide the nutrients obtained from carnivory. The elaborate mechanism that closes the trap is remarkably effective at capturing animals that trigger the trap. As many as $40 \%$ to $44 \%$ of traps that are triggered to close capture prey.

Acknowledgements: We thank Dr. Susan Verhoek of Lebanon Valley College for help in framing the study and constructive comments on the research. We would like to thank Rick Williams of RC Williams Company for designing the camera holder that played a crucial role in this research.

\section{References}

Adamec, L. 1997. Mineral nutrition of carnivorous plants: A review. The Botanical Review 63(6): 273-299.

Brown, W.H., and Sharp, L.W. 1910. The closing response in Dionaea. Bot. Gaz. 49: 290-302.

Lloyd, F.E. 1942. Carnivorous Plants. Chronica Botanica Co., Waltham, Mass.

Lichtner, F.T., and Williams, S.E. 1977. Prey capture and factors controlling trap narrowing in Dionaea (Droseraceae). American Journal of Botany 64(7): 881-886.

Hartmeyer, S.R.H., Hartmeyer, I., and Williams, S.E. 2019. Dionaea traps selectively allow small animals to escape. Carniv. P1. Newslett. 48(4):153-160.

Jones, F.M. 1923. The most wonderful plant in the world. Natural History 23(6): 589-596.

Juniper, B.E., Robins, R.J., and Joel, D.M. 1989. The Carnivorous Plants. Academic Press, London.

Pavlovic, A., and Mithöfer, A. 2019. Jasmonate signalling in carnivorous plants: copycat of plant defence mechanisms. Journal of Experimental Botany 70(13): 3379-3389.

Scherzer, S., Böhm, J., Krol, E., Shabala, L., Kreuzer, I., Larisch, C., Bemm, F., Al-Rasheid, K.A., Shabala, S., Rennenberg, H., Neher, E., and Hedrich, R. 2015. Calcium sensor kinase activates potassium uptake systems in gland cells of Venus flytraps. Proc. Natl. Acad. Sci. 112(23):73097314. 
Slack, A. 1980. Carnivorous Plants. MIT Press, Cambridge, Mass.

Williams, S.E. 1980. How Venus' flytraps catch spiders and ants. Carniv. Pl. Newslett. 9(3): 65, 75-78.

Williams, S.E., and Hartmeyer, S.R.H. 2017. Prey capture by Dionaea muscipula. A review of scientific literature with supplementary original research. Carniv. Pl. Newslett. 46(2): 44-61.

Williams, S.E., and Pickard, B.G. 1980. The role of action potentials in the control of capture movements of Drosera and Dionaea. In: Plant Growth Substances 1979 Edition: The Tenth International Conference on Plant Growth. Skoog, F. (ed.). pp. 470-480. Springer-Verlag, Berlin.

Williams, S.E., and Scholl, B. 2021a. Pollination of Dionaea muscipula, the Venus flytrap. Carniv. P1. Newslett. 50(1): 16-23.

Williams, S.E., and Scholl, B. 2021b. Using a trail camera for recording plant-insect interactions with Venus flytrap as an example. Carniv. P1. Newslett. 50(2): 66-72.
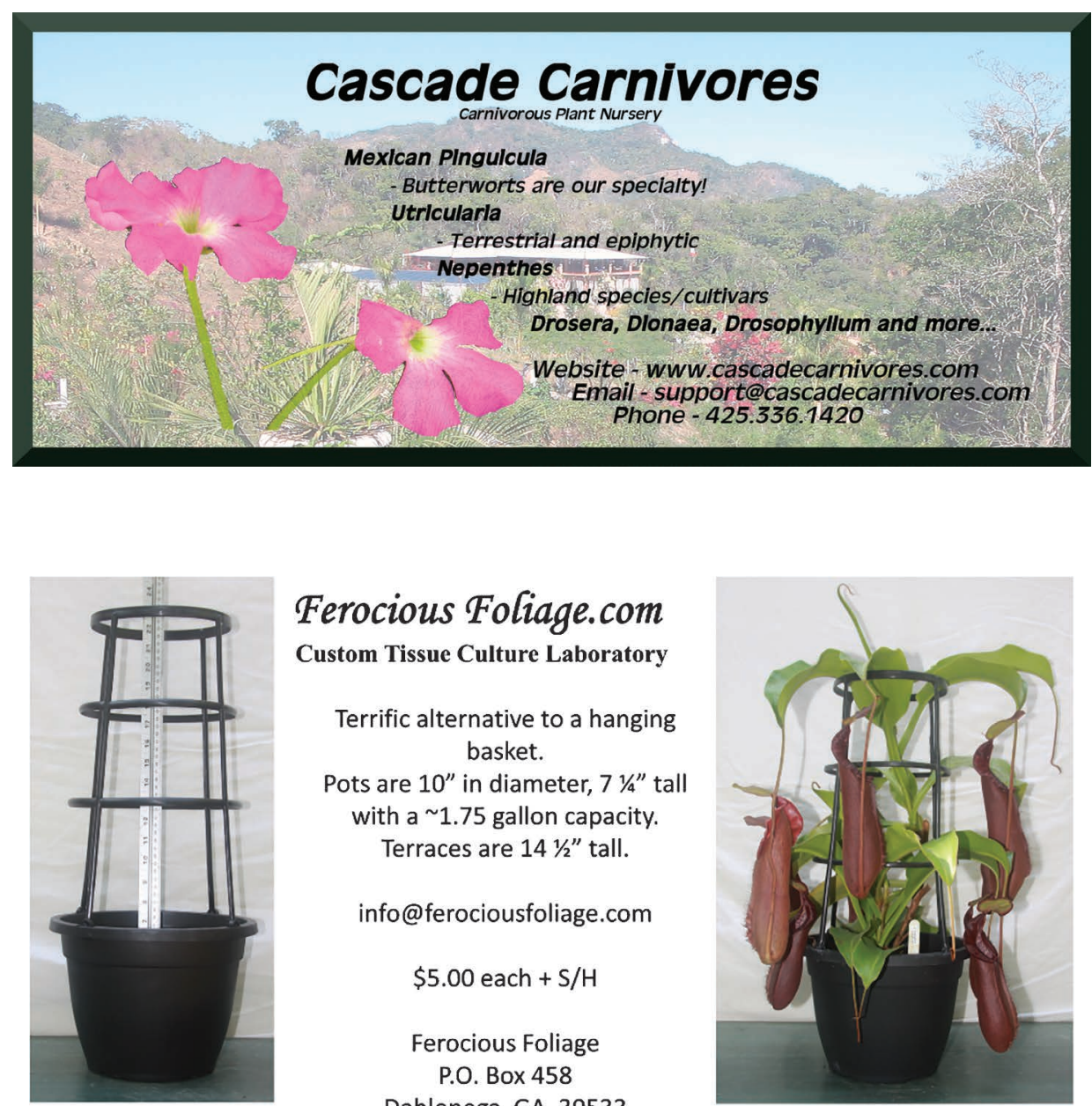

\section{Ferocious Foliage.com}

Custom Tissue Culture Laboratory

Terrific alternative to a hanging basket.

Pots are $10^{\prime \prime}$ in diameter, $71 / 4$ "tall with a 1.75 gallon capacity. Terraces are $141 / 2 "$ tall.

info@ferociousfoliage.com

$$
\begin{aligned}
& \$ 5.00 \text { each }+\mathrm{S} / \mathrm{H} \\
& \text { Ferocious Foliage } \\
& \text { P.O. Box } 458
\end{aligned}
$$

Dahlonega, GA 30533

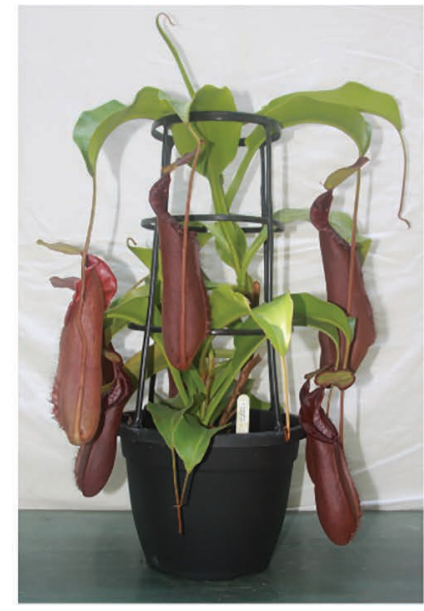

Plant not included 